Musées, Patrimoine et Culture scientifiques et techniques

$158 \mid 2015$

mars-avril 2015

\title{
Des étudiants traquent les préjugés au musée d'Ethnographie de Neuchâtel
}

\section{Bernard Knodel}

\section{OpenEdition \\ Journals}

Édition électronique

URL : http://journals.openedition.org/ocim/1491

DOI : 10.4000/ocim. 1491

ISSN : 2108-646X

Éditeur

OCIM

Édition imprimée

Date de publication : 1 mars 2015

Pagination : 13-18

ISSN : 0994-1908

Référence électronique

Bernard Knodel, «Des étudiants traquent les préjugés au musée d'Ethnographie de Neuchâtel », La Lettre de l'OCIM [En ligne], 158 | 2015, mis en ligne le 01 mars 2016, consulté le 22 avril 2019. URL: http://journals.openedition.org/ocim/1491; DOI : 10.4000/ocim.1491

Ce document a été généré automatiquement le 22 avril 2019

Tous droits réservés 


\title{
Des étudiants traquent les préjugés au musée d'Ethnographie de Neuchâtel
}

\author{
Bernard Knodel
}

L'affiche de Home sweet home mobilise certains éléments de l'exposition : le géranium emblématique des façades de chalets suisses, la notion de déracinement et la dominante blanche de l'espace d'exposition.

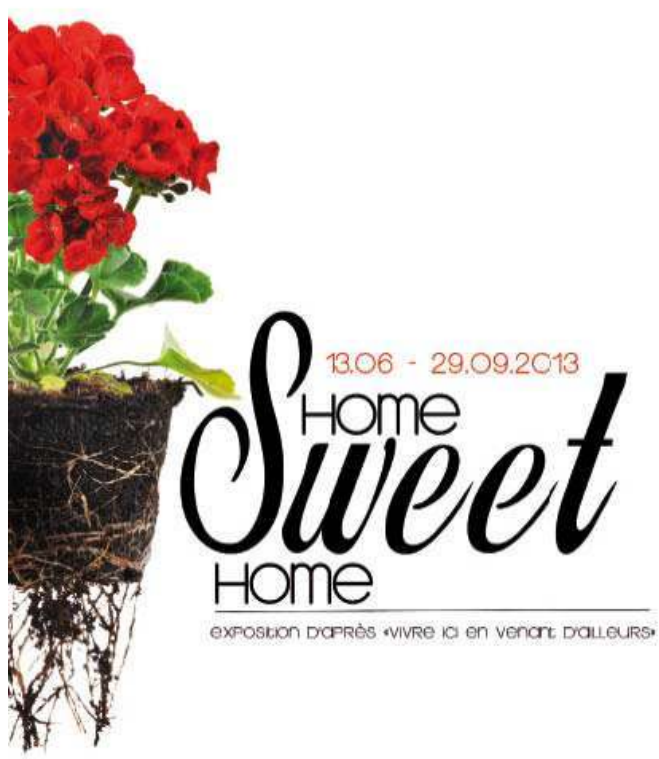

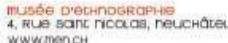

E. NEUCHÀTOI I 2013

(c) MEN/A. Gadenne 
1 Tous les deux ans, le musée d'Ethnographie de Neuchâtel (MEN) donne à un groupe d'étudiants de l'université de Neuchâtel l'opportunité de développer une exposition dans ses murs. Depuis les lectures et les recherches initiales jusqu'à la réalisation scénographique et au discours d'inauguration, cette expérience leur permet de découvrir les exigences du langage expographique, mais aussi de mettre en pratique quelques notions et savoir-faire indispensables à l'élaboration d'un dispositif, structuré d'abord conceptuellement puis spatialement.

2 À travers un retour sur la conception de l'exposition Home sweet home, il s'agit de présenter ici les enjeux didactiques et muséographiques d'un tel exercice, après avoir rappelé ses origines historiques et les conditions de sa mise en œuvre. En effet, son existence n'aurait pas été possible sans les liens étroits qui unissent le musée d'Ethnographie à l'université : la collaboration avec le récent Master en études muséales s'intensifie d'année en année, tandis que le MEN et l'Institut d'Ethnologie partagent leurs locaux et développent conjointement des projets d'exposition, de recherche et d'enseignement. Une telle complémentarité s'explique par l'histoire commune de ces deux institutions, qui, depuis de nombreuses années, a permis aux étudiants de l'université de Neuchâtel de participer, selon des modalités variées, aux expositions du musée.

\section{La participation régulière des étudiants}

Dès 1945, nommé simultanément professeur de géographie humaine à l'université et conservateur du musée d'Ethnographie, Jean Gabus profite de cette double responsabilité pour faire participer ses étudiants au montage des expositions temporaires annuelles. Dans le rapport de l'année 1948, il leur rend ainsi hommage : « Nous sommes heureux de remercier ici les étudiants qui nous ont aidé bénévolement, pendant plusieurs nuits consécutives, à monter l'exposition ${ }^{1}$. Tout en développant différents départements au sein du musée - la bibliothèque, l'ethnomusicologie, la photothèque ou encore l'atelier de décoration -, le conservateur reçoit régulièrement des stagiaires, et, dans le rapport annuel de 1963, annonce l'organisation de «travaux pratiques en ethnologie destinés à leur formation, dont bénéficient également les étudiants de l'université $»^{2}$.

Dans ce même rapport, commençant à employer couramment le terme d' « institut ", Jean Gabus insiste sur le lien essentiel entre les deux structures : « Il est évident qu'un Institut d'Ethnologie, avec le type d'enseignement qu'il comporte, ne saurait se passer du Musée, de ses collections, de son auditoire et de ses différents services, y compris les expériences muséographiques annuelles qui peuvent être faites - et qui sont faites - dans le bâtiment du Musée dynamique, pour chacune de nos expositions $»^{3}$. À la même période, il étend sa réflexion aux techniques d'expographie, s'inspirant notamment du théâtre pour forger le concept de musée-spectacle et, en 1965, il présente ses Principes esthétiques et préparation d'expositions didactiques dans deux numéros successifs de la revue internationale Museum. Mais la formation des étudiants et les travaux pratiques en ethnologie ne comprennent pas d'exercice de conception d'exposition, de scénographie, ou d'apprentissage d'un quelconque langage expographique. Se déroulant dans la réserve du musée, les travaux pratiques relèvent plutôt d'une initiation à l'étude, à l'inventaire, à la photographie et au conditionnement des collections muséales. La contribution effective des étudiants à la vie du musée consiste essentiellement en une participation au montage 
matériel des expositions, sous la direction de Walter Hugentobler, mais n'implique pas un accès de leur part à l'étape préalable de la conception.

5 L'année 1978 représente un tournant: après le départ de Jean Gabus s'ouvre pour le musée une période d'intérim. Pierre Centlivres, qui lui a succédé à la tête de l'Institut en 1974, et Jacques Hainard, alors chef de travaux à l'Institut, obtiennent des autorités politiques la responsabilité de l'exposition temporaire annuelle de 1979, Être nomade aujourd'hui. Cette exposition s'appuie sur la démarche d'analyse transversale et sur l'extension, à la société occidentale, des champs d'investigation de l'ethnologie telle qu'elle est enseignée à l'Institut. Mais surtout, pour la première fois, des secteurs entiers de l'exposition, sont conçus par des étudiants, dont Marc-Olivier Gonseth, qui fait là ses premières armes en concevant et réalisant une section consacrée aux «nomades du refus ".

L'extérieur de l'espace d'exposition situé devant l'accueil du musée évoque la façade d'un chalet suisse avec ses pots de géraniums sous les fenêtres et son paillasson Home sweet home. Cette façade contraste fortement avec l'espace intérieur d'un blanc immaculé.

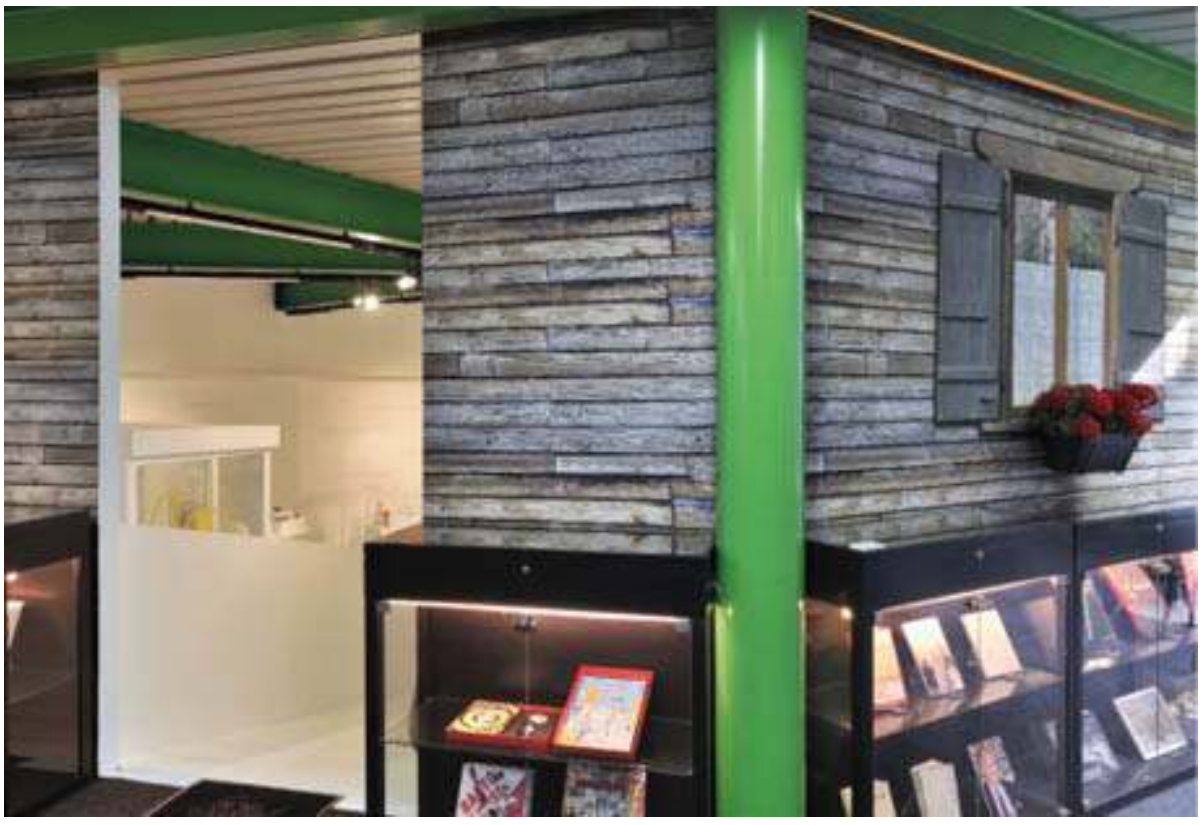

(c) MEN/A. Germond

\section{Du cours au travail pratique d'ethnomuséographie}

6 Nommé directeur du musée en 1980, Jacques Hainard donne annuellement à l'Institut un cours d'ethnomuséographie interrogeant les différents aspects de la notion de musée. Un semestre du cours est souvent consacré à la présentation et à l'analyse de l'exposition temporaire annuelle. À partir de 1986, les liens entre les deux institutions se renforcent par la construction du nouveau bâtiment, qui, en face de l'accueil du musée, présente un espace octogonal auquel on accède par une rampe. Il permet d'organiser des présentations ponctuelles de moindre envergure et de pallier ainsi la baisse de fréquentation du public entre deux expositions annuelles. Initialement, cet espace était destiné à la présentation de photographies, d'œuvres contemporaines ou encore des dons, legs et acquisitions récents ; il accueillait également des accrochages en lien avec les 
publications d'ouvrages des collections du musée. En 1997, Jacques Hainard crée le travail pratique d'ethnomuséographie tel qu'il existe aujourd'hui et décide de mettre périodiquement cet espace à disposition des étudiants. Lorsque Marc-Olivier Gonseth lui succède en 2006, l'aspect théorique de son enseignement à l'Institut est repris par Octave Debary dans un cours destiné aux étudiants de Bachelor. De son côté, le travail pratique destiné aux étudiants de Master est encadré par les conservateurs adjoints du musée : Olimpia Caligiuri, Yann Laville puis moi-même. Tous les deux ans, dix étudiants participent ainsi à la conception puis à la réalisation d'une exposition. L'exercice est l'occasion de développer tantôt une thématique en lien avec l'exposition annuelle, tantôt une problématique ethnologique ou muséologique, parfois en collaboration avec des partenaires externes au musée.

\section{Le TP d'ethnomuséographie et Neuchàtoi : un partenariat fécond}

7 L'exposition Home sweet home appartient à cette dernière catégorie, puisqu'il s'agit d'une collaboration entre les manifestations interculturelles de Neuchàtoi et le travail pratique d'ethnomuséographie. Les deux premières éditions de ces manifestations avaient déjà fait l'objet d'un partenariat fécond. En 2006, la thématique consacrée à l'identité neuchâteloise avait donné lieu à l'exposition Neuchensoi : réflexion sur les origines contrôlées. Puis la seconde édition, autour du thème de la jeunesse, avait inspiré l'exposition Météki? qui questionnait le regard porté sur les jeunes musulmans dans la société neuchâteloise. Pour la troisième édition des manifestations de Neuchàtoi, une nouvelle collaboration s'est imposée d'elle-même, d'autant plus que les ambitions visant à " promouvoir l'intégration de la diversité dans l'espace public, sans discrimination, et [à] donner confiance aux habitant-e-s du canton pour vivre ensemble dans le respect du pluralisme " ne pouvaient qu'interpeller les étudiants et le musée d'Ethnographie. La thématique retenue, "La diversité dans l'espace public », est aussi l'occasion de fêter les dix ans d'existence du projet «Vivre ici en venant d'ailleurs». Mené depuis 2002 par la journaliste indépendante Valérie Kernen, ce projet propose à des étrangers établis dans le canton de Neuchâtel de témoigner de leur parcours de vie et de faire part de leur regard sur la société et la culture Suisse. Chaque récit ou expérience donne lieu à un portrait écrit, photographique et oral, diffusé à une fréquence régulière par la presse de proximité et la radio régionale. Plus de 130 témoignages ont ainsi été rassemblés, recouvrant la diversité des quelques 150 nationalités présentes dans le canton. Tel est le matériau de base proposé à la petite équipe d'étudiants et stagiaires du travail pratique de l'année 2012-2013 formée d'Audrey Doyen, May Du, Agathe Gadenne, Diego Giannoni, Alexandre Lecoultre, Roman Lotocki et Margaux Peycelon. 
Vue de l'espace d'exposition octogonal depuis le haut de la rampe d'accès. L'espace consacré à l'apparence physique et vestimentaire avec le lave-linge et l'étendoir ; le texte d'introduction ; l'espace consacré au travail avec le bureau et l'espace relatif aux déplacements avec le chariot à bagages.

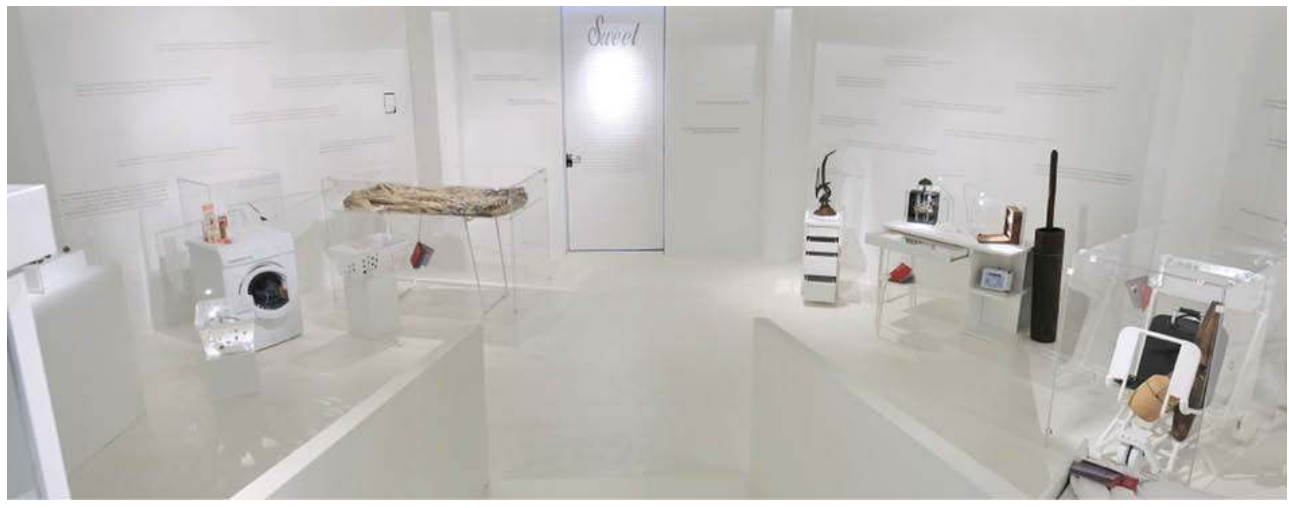

(c) MEN/A. Germond

\section{Home sweet home : dans la ligne de la muséologie de la rupture}

D'emblée le parti est clair : le but du TP d'ethnomuséographie n'est pas d'effectuer un simple accrochage des matériaux de "Vivre ici en venant d'ailleurs » en juxtaposant au mur une sélection de témoignages et de photographies des migrants. Il s'agit plutôt de poser un regard réellement critique et pertinent sur ce projet, afin de stimuler ensuite la réflexion du visiteur sur les problématiques qu'il véhicule. La conception de l'exposition « enseignée » dans le TP défend en effet une vision du musée comme lieu d'interrogation et de questionnement destiné à interpeller le visiteur. Ce principe relève de la «muséologie de la rupture » développée au musée d'Ethnographie de Neuchâtel, depuis les années 1980, par Jacques Hainard et Marc-Olivier Gonseth. Il postule, rappelons-le brièvement, que tout type d'exposition contient un propos - maîtrisé ou non - et que les expositions muséales ont vocation à être des discours subjectifs, des récits provoquant la réflexion du visiteur. Exposer signifie donc: « Raconter une histoire avec un début et une fin, troubler l'harmonie, susciter l'esprit critique, provoquer l'émotion dans la compréhension et la découverte de sens nouveaux [...] $»^{4}$. Le TP d'ethnomuséographie vise à sensibiliser les étudiants à ce langage et à les amener à en maîtriser quelques-uns des savoir-faire fondamentaux.

\section{Entre intuitions pertinentes et fausses pistes...}

Après la définition des deux sujets principaux de l'exposition, le témoignage et la migration, les premières séances - deux heures bimensuelles - sont consacrées à une présentation du musée et à une analyse de quelques-unes de ses expositions, réalisées pour certaines avec le TP d'ethnomuséographie, et dont les thématiques ou les artifices scénographiques peuvent alimenter la conception de l'exposition future. Parallèlement, un calendrier commun est élaboré à partir d'une présentation des différentes phases de conception et de réalisation d'une exposition distinguées par Marc-Olivier Gonseth ${ }^{5}$. 
Venue expliquer sa démarche aux étudiants, la journaliste Valérie Kernen leur confirme qu'ils ont carte blanche pour utiliser son travail et ses matériaux comme bon leur semble.

Afin de rester fidèles à la transversalité des expositions du musée d'Ethnographie, les étudiants sont donc invités à nourrir leur regard sur les témoignages de "Vivre ici en venant d'ailleurs » le plus largement possible, en parcourant des ouvrages fictionnels et scientifiques, en explorant les médias, en confrontant leurs préjugés, et en partageant leurs intuitions. Plusieurs pistes sont ainsi abordées telles que la possibilité de développer l'environnement scénographique de l'exposition en s'inspirant des lieux de témoignage de notre monde contemporain: le confessionnal, le plateau de télévision, le palais de justice... Une autre alternative met plutôt l'accent sur la question d'un monde en constant mouvement où non seulement les hommes, mais également les choses, se déplacent. L'idée serait de "faire témoigner» des "entités migrantes »: une spécialité culinaire (hamburger, pizza...), un animal (on pense par exemple à la réintroduction de l'ours dans les régions alpines ou à l'apparition, en Europe, du frelon asiatique...), une langue, un vêtement...

11 Après ce tour d'horizon, entraînant parfois des visualisations scénographiques délirantes en regard du peu d'espace et de moyens disponibles, les étudiants concepteurs se concentrent à nouveau sur les matériaux mis à leur disposition, tout en gardant à l'esprit quelques-unes des intuitions premières et des pistes de réflexion déjà évoquées. Il semble alors important de remettre en question le rôle des frontières pour déterminer ce qui migre ou ce qui devient étranger, les phénomènes migratoires gagnant à être envisagés par des perspectives transnationales ou d'hybridité et de métissage ; ainsi, le proche peut parfois sembler bien étranger; inversement, la question de la nationalité n'est pas toujours pertinente pour penser l'altérité ; enfin, les parcours individuels sont beaucoup plus riches et complexes que ne l'implique la vision simpliste véhiculée par le sens commun, les médias ou les préjugés. Loin des récupérations politiques, des réponses partisanes et des jugements à l'emporte-pièce souvent générés par ce sujet de société qu'est la migration, il s'agit plutôt de révéler la complexité de ses problématiques en suscitant une réflexion sur les rapports entre les cultures à l'ère de la globalisation. Dans cette dynamique, le musée d'Ethnographie de Neuchâtel ne serait-il pas un home sweet home particulièrement propice à une telle sensibilisation? 
La partie famille avec, au premier plan, les passeports puis les crânes issus d'un coffre-reliquaire du Gabon dédié au culte des ancêtres et, inscrite au mur, la phrase : « Il est important d'être en lien avec ses ancêtres, de savoir d'où l'on vient » (extrait du témoignage d'Enver, originaire du Kosovo).

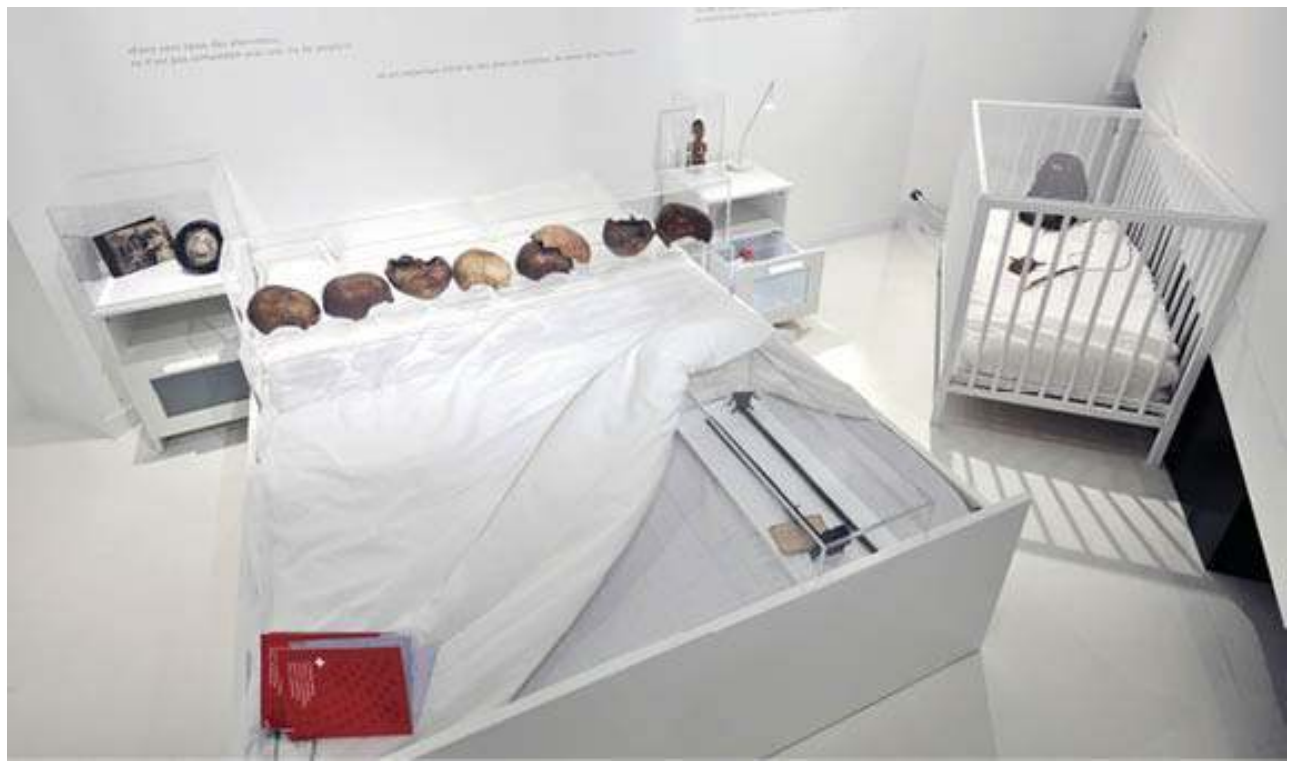

(c) MEN/A. Germond

\section{Sélectionner : une démarche constructive}

Les lectures et les écoutes des témoignages font progressivement émerger des thématiques récurrentes telles que l'alimentation, l'arrivée en Suisse, le travail, la formation, la vie familiale, les motivations du départ, la langue, les différences culturelles, l'intégration... Se livrant à une sélection drastique, l'équipe parvient à réduire ces thématiques au nombre de six : la communication, les croyances, l'apparence physique et vestimentaire, le travail, les déplacements et, enfin, la famille. Chacune de ces thématiques permet de ne pas stigmatiser telle ou telle catégorie de migrants, tout comme les citations extraites des témoignages, destinées à figurer sur les murs de l'espace d'exposition octogonal. Ainsi les allusions à une nationalité ou à une ère culturelle déterminée ne doivent pas apparaître de manière trop évidente. Bien qu'extrêmement récurrente, la thématique de l'alimentation est ainsi éliminée car les allusions à des spécialités culinaires précises sont trop souvent présentes dans les témoignages. Les citations telles que «On commence par la salade russe, vraie salade russe, pas comme on la connaît ici, parce que normalement, toujours, la salade russe est préparée avec de la viande de bœuf» (Pavel, Russie, novembre 2012) ou celles dans lesquelles apparait explicitement un pays ou une nationalité, comme: "Au Pérou, la religion est transmise aux enfants par leur mère ; j'ai 83,2\% de sang chinois mais nous avons hérité de la confession de notre grand-mère maternelle qui était Péruvienne (sic) » (Irma, Pérou) sont écartées. La citation sélectionnée doit pouvoir être lue par n’importe quel visiteur sans qu'il préjuge de l'origine du locuteur. Au contraire, il est impératif qu'il puisse s'identifier au propos jusqu'à se dire : « Moi aussi, j'ai le même regard sur cela! ». Les citations sont donc inscrites sur les murs, et leurs références - prénom, nationalité, date et titre du témoignage - reléguées sur le cartel. Pour accentuer encore l'effet de 
diversité, une citation externe au projet, d'un anthropologue ou d'un écrivain, est intégrée aux témoignages afin d'élargir le regard porté sur chacune des thématiques.

\section{Un dispositif simple pour complexifier le propos}

Pour la mise en scène proprement dite, dont la réalisation est encadrée par Nicolas Sjöstedt, l'équipe de conception part d'un dispositif assez simple, susceptible à la fois de signifier la question abordée - de faire signe - et de servir de support ou de socle aux objets des collections. Plutôt que d'inscrire un titre au mur pour chacune des sections, l'équipe de conception décide de structurer l'espace avec des objets banals du quotidien appartenant soit à la sphère privée, soit à la sphère publique. Chacun de ces six objets dits «balises » est placé devant le pan de mur de l'octogone où sont inscrites les citations correspondant à la thématique qu'il évoque. Une cabine téléphonique est choisie pour le thème de la communication, un autel pour les croyances, un lave-linge et un étendoir pour l'apparence vestimentaire et physique, un bureau pour le travail, un chariot à bagages pour les déplacements, un lit double et un berceau pour la famille.

La partie sur la communication avec la cabine téléphonique comme objet « balise ».

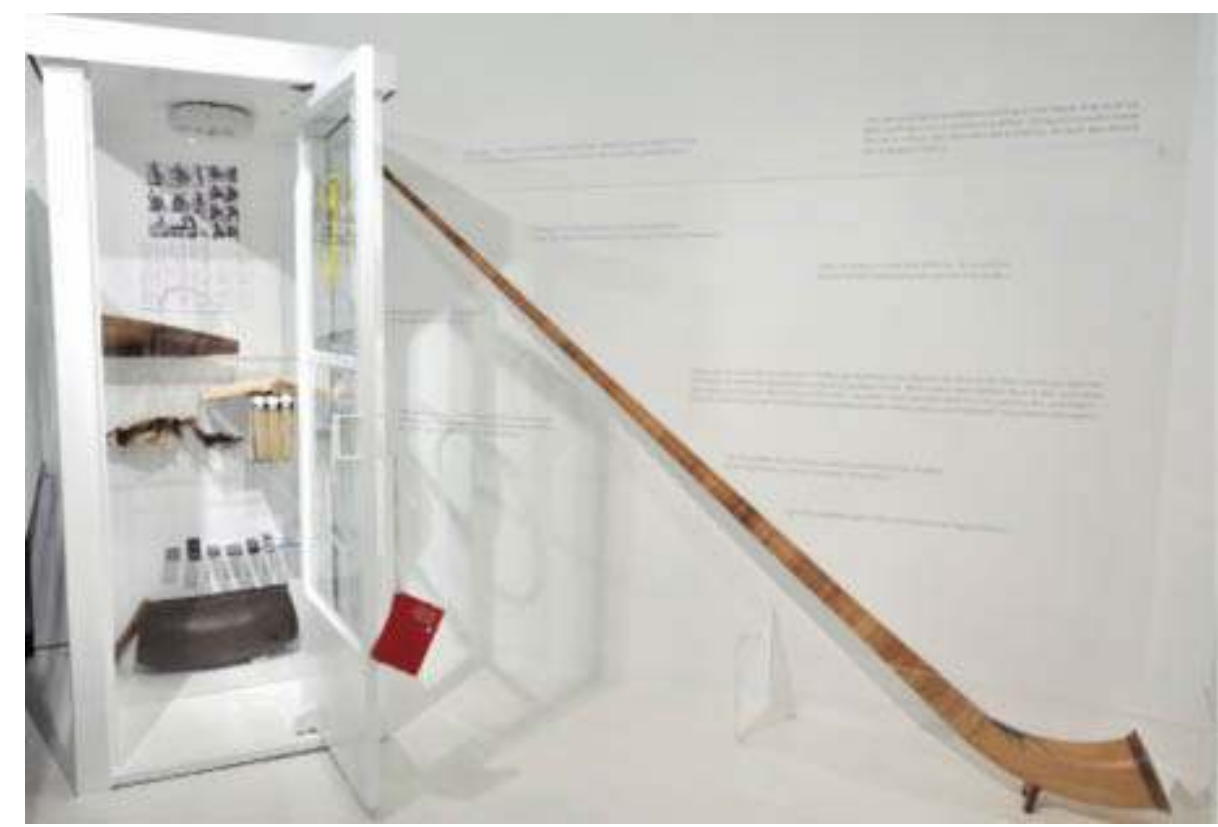

(C) MEN/A. Germond

Parallèlement, les étudiants-concepteurs effectuent une recherche dans les collections du musée, par le biais de l'inventaire informatique. En exploitant les artefacts provenant des cinq continents conservés dans les réserves, ils tentent de déconstruire, ou tout du moins de dépasser, les concepts d'« ici » et d'« ailleurs » envisagés comme une dichotomie trop stricte et figée, afin de restituer à la thématique de la migration toute sa complexité. Ainsi le choix des objets des collections se trouve déterminé en fonction de deux axes cardinaux : altérité extrême/ "suissitude » et local/global. Lors des séances collégiales, les étudiants soumettent aux autres membres de l'équipe de conception le fruit de leur investigation, afin d'en tester la lisibilité. L'équipe peut ainsi vérifier l'adéquation des objets aux axes de sélection énoncés. Par ailleurs, si les collections du musée ne 
permettent pas d'aborder certaines problématiques présentes dans les témoignages, les concepteurs décident de les emprunter dans d'autres institutions muséales, chez des particuliers ou de les acquérir. Ainsi, une lotion auto-bronzante et une préparation pour éclaircir la peau sont achetées dans des commerces neuchâtelois pour évoquer la récurrence et l'ambivalence de la couleur de peau ressentie comme un facteur d'appartenance ou de rejet.

La partie consacrée du travail avec le bureau comme objet « balise ».

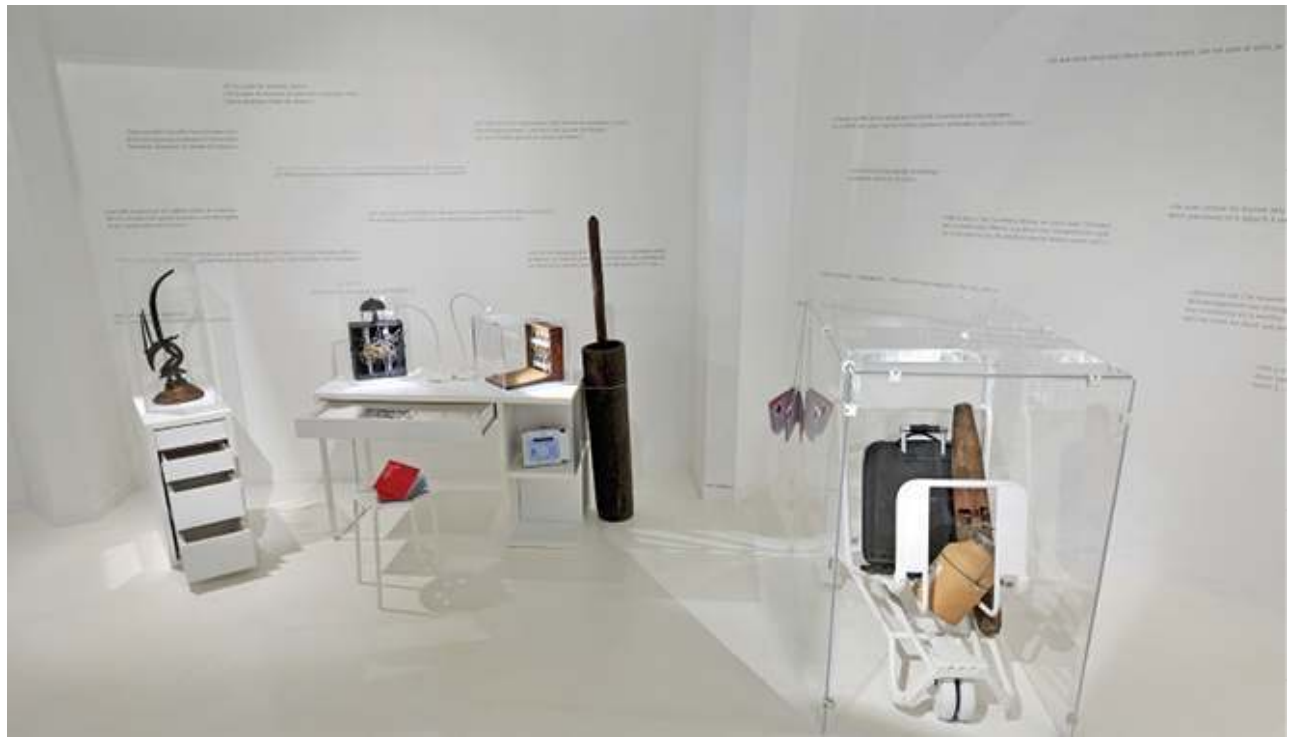

(C) MEN/A. Germond

\section{L'épreuve de l'espace}

$\mathrm{Au}$ fur et à mesure que s'approche le moment de l'intervention dans l'espace, les choix se concrétisent et s'arrêtent. Au cours du mois de février, l'équipe de conception choisit le titre définitif de l'exposition Home sweet home qui, dès lors, sera annoncé dans la programmation du festival Neuchàtoi 2013 et dans la communication du musée. L'enveloppe scénographique se définit plus précisément : un espace extérieur évoque une façade de chalet suisse et contraste fortement avec un intérieur entièrement blanc et très lumineux dans lequel se fondent les objets balises recouverts de blanc. Hormis l'avantage d'un coût modeste, le choix d'une dominante blanche dans l'espace d'exposition cumule plusieurs avantages. D'un point de vue symbolique, le blanc peut être lu comme une allusion à la neutralité et à la propreté suisse, rappelant la perception stéréotypée d'un pays "à l'abri des conflits", "propre en ordre " et où "rien ne dépasse ", souvent présente dans les témoignages des migrants. Cette blancheur évoque également un espace de projection ou de représentation : celui du musée, conçu comme un miroir que la société se tend à elle-même, comme un lieu idéal pour mener une réflexion et débattre à l'écart des préjugés véhiculés par le sens commun et les médias. Enfin, d'un point de vue scénographique, la neutralisation, par le blanc, de l'environnement d'exposition (mur et sol) et des objets balises réduits au rang de signe (cabine téléphonique, autel, machine à laver...) permet de centrer l'attention du visiteur sur les objets exposés et sur les citations qui se détachent en gris sur les murs immaculés. 

d'échapper au critère de la nationalité, jugé d'emblée trop réducteur pour analyser le phénomène complexe des processus migratoires et pour mesurer le degré d'étrangeté, le modèle du passeport suisse est choisi comme support. Ainsi, chaque secteur comporte des passeports qui contiennent les «fiches d'identités » des objets exposés, s'inspirant des rubriques du document officiel original. Les deux dernières pages rassemblent les citations affichées au mur de la section correspondante en mentionnant les noms et prénoms, les nationalités, les titres des articles et les dates des témoignages.

Cette présentation de la genèse de Home sweet home met l'accent sur quelques aspects essentiels de la conception de l'exposition, mais passe sous silence beaucoup d'autres expériences transmises aux étudiants: l'obtention de prêts auprès de particuliers ou d'institutions partenaires, l'écriture des textes de l'exposition, la gestion des délais et du budget, la recherche dans la base de données du musée, ou encore la communication auprès du public et des médias. Mais les savoir-faire et la maîtrise des langages expographiques ne s'acquièrent que par la multiplication des expériences; comme le sait tout concepteur d'exposition, il n'existe pas de méthode systématique ou de recette infaillible pour aboutir à une exposition de qualité et chaque projet recèle sa part d'inconnu et d'imprévu. À un autre niveau, chaque travail pratique est donc aussi, pour le conservateur qui encadre les étudiants, une occasion précieuse et passionnante de remettre en question sa propre pratique, de bousculer ses habitudes et ses théories et de redécouvrir la richesse formidable des différentes facettes du métier de muséographe.

La prochaine exposition réalisée par les étudiants $\mathrm{du}$ travail pratique d'ethnomuséographie sera consacrée à la mort et aura lieu du 27 juin 2015 au 3 janvier 2016 au musée d'Ethnographie de Neuchâtel.

\section{Extrait d'un courrier électronique adressé par l'un des étudiants au reste de l'équipe de conception à propos des artefacts choisis pour la section consacrée au travail}

19 «En parallèle l'idée d'un nouvel objet évoquant la suissitude est apparue. C'est un almanach: "Le véritable messager boiteux de Neuchâtel» (le nom est génial!). Ces ouvrages traitent par exemple du calendrier des activités agricoles (parfait pour la section travail). Je vous indique le numéro que j'ai consulté pour voir à quoi ça ressemble : http://doc.rero.ch/record/12448?ln=fr (regardez page 4 du numéro de 1960), perso j'aime bien l'icono de couverture style «traditionnelle et authentique » qui renvoie à un idéal type de Neuchâtel (lac, vignes, château, travaux agricoles...).

À voir... si l'idée vous plait.

21 Pour revenir sur l'avancement de la section, aborder le travail dans son sens global paraissait trop large. L'idée de réfléchir au thème de la rythmique dans le cadre du travail est apparue (saisonnalité, cadence, productivité, horaires, répétition de mouvements et de pratiques...). Cette idée semble bien coller et articuler les objets qui seront présentés :

22 - le pilon à coca (altérité) évoque une rythmique, la répétition du mouvement. L'intérêt de l'objet est aussi ce qu'il sert à broyer : la coca et son pouvoir stimulant renvoient à la productivité et à la performance, deux choses particulièrement valorisées dans le travail aujourd'hui ; 
23 - la pointeuse (globalisation) renvoie aux horaires et à la nécessité de les respecter, c'est aussi la répétition d'une activité enregistrée jour après jour ;

24 - l'almanach neuchâtelois (local/suissitude) évoque la saisonnalité, les cycles dans le travail ;

- mécanismes de montre (local/suissitude): engrenage, répétition infinie du geste, précision... De plus l'image de qualité de l'horlogerie suisse ramène à la performance (qualité et quantité) demandée dans le travail ;

26 - permis de travail : question du contrôle mais aussi de la durée du travail (permis valable tant de temps).

27 Il me semble que ces objets ne poseront pas trop de problème pour l'installation et s'articuleront même bien autour de notre objet balise.

28 Il y a toujours l'idée de l'album Suchard avec l'image «mon cher pays » qui reste en suspens...»

\section{BIBLIOGRAPHIE}

Gabus, J. Musée d'ethnographie, Bibliothèques et Musées de la Ville de Neuchâtel 1948, nº 2, 1949, pp. 37-52.

Gabus, J. Musée d'ethnographie et Institut d'ethnologie, Bibliothèques et Musées de la Ville de Neuchâtel 1963, n 4, 1964, pp. 64-129.

Gabus, J. Principes esthétiques et préparation des expositions didactiques, Museum, $\mathrm{n}^{\circ}$ XVIII, 1965, 1 pp. 3-59 et 2 pp. 65-97.

Gonseth, M.-O. Un atelier expographique, in Gonseth, M.-O., Hainard, J. et Kaehr, R. Cent ans d'ethnographie sur la colline de Saint-Nicolas 1904-2004, Neuchâtel : Musée d'ethnographie, 2005, pp. 375-394.

Hainard, J. Pour une muséologie de la rupture, in Desvallées, A., De Bary, M.-O. et Wasserman, F. Vagues. Une anthologie de la nouvelle muséologie. Mâcon et Savigny-le-Temple : Éditions W. et M.N.E.S. t. 2, 1994, pp. 531-539.

\section{NOTES}

1. Gabus, J. Musée d'ethnographie, Bibliothèques et Musées de la Ville de Neuchâtel $1948, \mathrm{n}^{\circ} 2$, 1949, p. 49.

2. Gabus, J. Musée d'ethnographie et Institut d'ethnologie, Bibliothèques et Musées de la Ville de Neuchâtel 1963, n4, 1964, p. 83.

3. Gabus, J. Musée d'ethnographie et Institut d'ethnologie, Bibliothèques et Musées de la Ville de Neuchâtel 1963, n4, 1964, p. 65. 
4. Hainard, J. Pour une muséologie de la rupture, in Desvallées, A., De Bary, M.-O. et Wasserman, F. Vagues. Une anthologie de la nouvelle muséologie. Mâcon et Savigny-le-Temple : Éditions W. et M.N.E.S. t. 2, 1994, p. 538.

5. Gonseth, M.-O. Un atelier expographique, in Gonseth, M.-O., Hainard, J. et Kaehr, R. Cent ans d'ethnographie sur la colline de Saint-Nicolas 1904-2004. Neuchâtel : Musée d'ethnographie, 2005 , p. 392.

\section{RÉSUMÉS}

Permettre aux étudiants de mettre en pratique les éléments théoriques enseignés à l'université, tel est l'objectif principal de l'exercice de conception d'exposition, de scénographie, d'apprentissage et de sensibilisation au langage expographique organisé et encadré depuis près de vingt cinq ans par le musée d'Ethnographie de Neuchâtel.

\section{INDEX}

Mots-clés : préjugé, ethnographie

\section{AUTEUR}

\section{BERNARD KNODEL}

conservateur-adjoint au musée d'Ethnographie de Neuchâtel et responsable du travail pratique d'ethnomuséographie

bernard.knodel@ne.ch 